\title{
PROTOKOLL DER 64. GENERALVERSAMMLUNG DER VEREINIGUNG ÖSTERREICHISCHER BIBLIOTHEKARINNEN UND BIBLIOTHEKARE
}

\author{
Datum: 12. September 2019, 16:15 Uhr \\ Ort: Messe Congress Graz \\ Vorsitz: Werner Schlacher / Bruno Bauer \\ Protokoll: Markus Lackner
}

\section{TOP 1: Begrüßung und Feststellung der Beschlussfähigkeit}

Schlacher begrüßt die Anwesenden zur 64. Generalversammlung und stellt die Beschlussfähigkeit fest. Die Moderation dieser Generalversammlung ist seine letzte Amtshandlung und er freut sich, das Amt im Laufe dieser Veranstaltung in jüngere Hände legen zu dürfen.

Der Tagesordnungspunkt 8 wird durch einen Vortrag der PresidentElect der IFLA, Barbara Lison, bereichert.

TOP 2: Genehmigung des Protokolls der 63. Generalversammlung der VÖB der Funktionsperiode 2015-2017 vom 14.09.2017

Es gibt keine Anmerkungen zum Protokoll der 63. Generalversammlung.

\section{TOP 3: Tätigkeitsbericht über die vergangene Funktionsperiode}

Schlacher stellt den Antrag, den Tätigkeitsbericht, wie er auf der Homepage verfügbar ist, anzunehmen. Der Antrag wurde einstimmig ohne Gegenstimmen angenommen.

Schlacher dankt den Kommissionsvorsitzenden für die Erstellung ihrer Berichte. Das Angebot von Gerald Leitner, den IFLA Kongress 2021 in Österreich durchzuführen, konnte leider nicht angenommen werden, da die Vorbereitungszeit zu kurzfristig gewesen wäre. Dies war jedoch der Ausgangspunkt zu weiteren Gesprächen zwischen VÖB und BVÖ über eine Annäherung der Verbände. Im ersten Schritt hat man sich auf die gemeinsame Abhaltung eines Bibliothekskongress geeinigt. Wünsche über eine tiefergehende Zusammenarbeit wurden mit Christian Jahl besprochen. Da Österreich in internationalen Gremien stark unterpräsentiert ist, wäre ein gemeinsames Einbringen durch beide Verbände wünschenswert. Diese Annäherung war wesentlicher Teil der letzten 2 Jahre. 
Erwähnenswert ist auch die InetBib-ODOK Veranstaltung, die von 21. bis 23. Februar 2018 in Wien stattgefunden hat. Sie war nicht nur eine inhaltliche Bereicherung, sondern auch ein finanzieller Gewinn. Es ist daher umso bedauerlicher, dass wir die ODOK als Veranstaltungsserie nicht weiterführen werden. Das hängt damit zusammen, dass Kollegin Dr. Eveline Pipp, die diese Veranstaltungsreihe maßgeblich organisiert hat, verstorben ist. Durch die gemeinsame InetBib-ODOK gab es den Versuch, dem eine Zukunft zu geben, dies kann aber leider nicht weitergeführt werden. Die Einnahmen dieser Veranstaltung entfallen somit. Weitere Tagung war die DACHS-Tagung, welche bei der nächsten Durchführung in der Schweiz stattfinden wird.

Schlacher dankt Ute Wödl für die Zurverfügungstellung der Räumlichkeiten an der AK für die Präsidiums- und Vorstandssitzungen. Schlacher dankt auch seinen Vizepräsidentinnen sowie Lackner, Kreinz und Druschowitz für die gute Zusammenarbeit.

\section{TOP 4: Kassenbericht und Entlastung des Präsidiums}

Kreinz präsentiert den Finanzbericht, der im Tätigkeitsbericht enthalten ist. Im ordentlichen Haushalt gibt es mehr Ausgaben als Einnahmen. Viele Jahre war das kein Problem, da dieser negative Betrag durch Gewinne aus den Veranstaltungen kompensiert werden konnte. Bei den Bibliothekartagen gibt es immer weniger Firmen und Teilnehmer. Der Wegfall der ODOK verschärft diese Situation. Um das ordentliche Budget ausgeglichen zu gestalten, wurde versucht, alle Ausgaben zu optimieren. Weiters muss der Mitgliedsbeitrag erhöht werden.

Kreinz stellt daher den Antrag auf Erhöhung der Mitgliedsbeiträge auf EUR 40,- für Vollzahlerlnnen und EUR 20,- für ermäßigte Zahlerlnnen. Dadurch kann das neue Präsidium mit einem ausgeglichenen Budget starten. Kreinz dankt allen für die pünktliche Zahlung der Mitgliedsbeiträge.

Um mit der Erhöhung länger auszukommen, stellt Schlacher den Antrag, die Mitgliedsbeiträge für VollzahlerInnen auf EUR 45,- und für ermäßigte Zahlerlnnen auf EUR 25,- zu erhöhen.

Bericht der Kassenrevisoren: Bei den Prüfungen durch die Kassenrevisoren wurden immer alle Belege vorgelegt und es wurden keine Probleme festgestellt. Der Kassier konnte alle Fragen beantworten.

Offenbacher stellt den Antrag auf Entlastung des Kassiers und des Präsidiums. Der Antrag wird einstimmig angenommen.

Bezüglich der Mitgliedsbeitragserhöhung wird vorgeschlagen, über die Erhöhung für VollzahlerInnen und ermäßigten Zahlerlnnen getrennt abzu- 
stimmen. 51 sind für eine getrennte Abstimmung, 4 dagegen und 3 enthalten sich.

Schlacher formuliert seinen Antrag neu: Wer ist für eine Erhöhung des Mitgliedsbeitrages für Vollzahlerlnnen auf EUR 45,-?

Der Antrag wird mit einer Stimmenthaltung, ohne Gegenstimme angenommen.

Schlacher formuliert seinen Antrag neu: Wer ist für eine Erhöhung des Mitgliedsbeitrages für ermäßigte ZahlerInnen auf EUR 25,-?

Der Antrag wird mit 5 Stimmenthaltung und 8 Gegenstimmen angenommen.

\section{TOP 5: Wahl der Kassenrevisor/inn/en}

Schlacher stellt den Antrag, Kollegen Martin Kreinz und Kollegin Ute Wödl als Kassenrevisoren zu bestellen.

Der Antrag wird mit einer Enthaltung angenommen.

\section{TOP 6: Beschlussfassung über die Verleihung von Ehrungen}

Die Beschlussfassung fand bereits in der a.o. Generalversammlung statt.

TOP 7: Beschlussfassung über eingegangene Anträge

Es sind keine weiteren Anträge eingegangen.

TOP 7a: Antrag auf Erhöhung des Mitgliedsbeitrages auf EUR 40,- für Vollzahler bzw. auf EUR 20,- für ermäßigte Zahler (Beauftragung des Präsidiums in der 2. Vorstandssitzung der Funktionsperiode 2017-2019)

Wurde bereits unter TOP 4 behandelt.

\section{TOP 8: Allfälliges}

Die President-Elect der IFLA, Barbara Lison, berichtet über die IFLA-Survey.

TOP 9: Ergebnisse der Wahl der Präsidentin/des Präsidenten und ihrer/ seiner Stellvertreter/innen für die Funktionsperiode 2019-2021

Lackner verliest das Wahlprotokoll: Bis 12 Uhr gab es die Möglichkeit den VÖB-Vorstand und das VÖB-Präsidium zu wählen. Im Anschluss wurde die 
Wahlurne durch den Wahlleiter Luzer abgeholt und die Stimmen gemeinsam mit Druschowitz ausgezählt.

\section{Ergebnis der Präsidiumswahl:}

Es wurden 179 Stimmzettel abgegeben. Für den Wahlvorschlag stimmten 170 Mitglieder.

Der neu gewählte Präsident Bruno Bauer und die beiden Stellvertrreterinnen Pamela Stückler und Eva Ramminger nehmen die Wahl an.

TOP 10: Ergebnisse der Wahl für den Vorstand der VÖB für die Funktionsperiode 2019-2021

Lackner verliest das Ergebnis der Vorstandswahlen:

\begin{tabular}{|l|l|}
\hline Susanne Blumesberger & 122 Stimmen \\
\hline Markus Lackner & 101 Stimmen \\
\hline Andreas Ferus & 89 Stimmen \\
\hline Marion Kaufer & 84 Stimmen \\
\hline Martin Kreinz & 79 Stimmen \\
\hline Markus Stumpf & 75 Stimmen \\
\hline Ute Wödl & 71 Stimmen \\
\hline Christa Müller & 66 Stimmen \\
\hline Patrick Danowski & 63 Stimmen \\
\hline Wolfgang Hamedinger & 62 Stimmen \\
\hline Ortwin Heim & 42 Stimmen \\
\hline Marian Miehl & 41 Stimmen \\
\hline Bernhard Kurz & 35 Stimmen \\
\hline Lorenz Mikoletzky & 22 Stimmen \\
\hline
\end{tabular}

Luzer gratuliert allen gewählten Kolleginnen und Kollegen.

Der neu gewählte Präsident der VÖB, Bruno Bauer, dankt allen, die bisher in Präsidium und Vorstand in der VÖB tätig waren. Sein Dank gilt auch Pamela Stückler und Eva Ramminger, für ihre Bereitschaft, die in der kommenden Präsidiumsperiode 2019 bis 2021 als Stellvertreterinnen fungieren 
zu wollen, sowie allen, insbesondere auch den neuen und jungen Kolleginnen und Kollegen, die im künftigen Präsidium mitarbeiten werden.

Ein besonderer Dank gebührt auch dem Altpräsidenten Werner Schlacher und den weiteren Präsidiumsmitgliedern für die bisherige und auch für die Zukunft zugesagte Unterstützung des neuen Präsidiums. Für die Bereitschaft, als kooptierte Mitglieder im neuen Präsidium für Informationen zur Verfügung zu stehen und damit ihr wertvolles Wissen weiterhin uneingeschränkt der VÖB zur Verfügung zu stellen, schuldet die VÖB Schlacher und Seissl großen Dank.

Durch die laut Statuten vorgesehene Mitgliedschaft der AG- und Kommissionsvorsitzenden im Vorstand, wird es möglich sein, dass alle Kandidatinnen und Kandidaten aus der Wahlliste nachrücken können.

\section{TOP 11: Änderung der Statuten (Anpassung des Vereinssitzes)}

Bauer stellt den Antrag, den Vereinssitz nach Wien zu transferieren. Der Antrag wird einstimmig, ohne Enthaltungen angenommen.

\section{TOP 12: Schlussworte des Präsidenten}

Bauer bedankt sich bei allen Anwesenden für das in das neue Präsidium gesetzte Vertrauen und weist darauf hin, dass dessen Mltglieder jederzeit für Fragen und Anliegen zur Verfügung stehen.

Er bedankt sich auch bei allen, die zum guten Gelingen des 34. Österreichischen Bibliothekartages in Graz beigetragen haben und weist darauf hin, dass der Schwerpunkt der kommenden Funktionsperiode der 1. Österreichische Bibliothekskongress in Innsbruck im April 2021 sein wird.

Um 17.45 Uhr schließt Bauer die Generalversammlung und wünscht allen eine schöne Zeit am bevorstehenden Festabend. 